\title{
ESTUDO DO EQUILÍBRIO DE FASES DE SISTEMAS COMPOSTOS POR $\beta$-CARIOFILENO, EUGENOL, ETANOL E ÁGUA APLICADO AO FRACIONAMENTO DE ÓLEO ESSENCIAL DE CRAVO
}

\author{
C. C. KOSHIMA, L. L. VENÂNCIO, K. T. NAKAMOTO, T. K. UMEDA, K. K. ARACAVA e C. E. \\ C. RODRIGUES
}
Universidade de São Paulo, Faculdade de Zootecnia e Engenharia de Alimentos, Departamento de Engenharia de Alimentos E-mail para contato: Cristina.chiyoda@usp.br

\begin{abstract}
RESUMO - Óleos essenciais apresentam em sua composição hidrocarbonetos terpênicos e terpenos oxigenados. Estes últimos são mais estáveis e aromatizantes, se comparados aos hidrocarbonetos que, quando submetidos ao calor ou oxigênio sofrem degradação resultando na perda de qualidade do óleo. Para melhorar a qualidade dos óleos, a desterpenação propõe o enriquecimento da mistura nos produtos oxigenados através da remoção parcial dos compostos terpênicos. O estudo do equilíbrio de fases fornece as informações essenciais para o correto projeto e otimização dos processos de separação. Dados experimentais de equilíbrio líquido-líquido de sistemas contendo $\beta$-cariofileno, eugenol e solvente etanol hidratado foram correlacionados utilizando os modelos NRTL e UNIQUAC, os quais apresentaram desempenho similar com valores de desvio global próximos a $0,5 \%$ entre as composições experimentais e calculadas. Verificou-se que o eugenol apresentou o maior coeficiente de partição e o aumento da hidratação do solvente ocasionou uma menor migração dos compostos do óleo essencial para a fase solvente.
\end{abstract}

\section{INTRODUÇÃO}

O Brasil apresenta um grande potencial de obtenção de diferentes produtos com capacidades aromatizantes e funcionais, fato este extremamente interessante para o mercado de óleos essenciais. No entanto, poucas foram as iniciativas para o melhor aproveitamento destes recursos, o que levou o país para a situação em que se encontra, um exportador de matérias-primas para síntese de produtos com alto valor agregado (Bizzo et al., 2009).

Óleos essenciais são produtos de origem vegetal constituídos por diversos componentes químicos diferentes que incluem majoritariamente compostos voláteis, como hidrocarbonetos terpênicos e compostos oxigenados, e minoritariamente compostos não voláteis como pigmentos, ceras, resinas e flavonóides (Bizzo et al., 2009; Diaz et al., 2005).

Com relação a fração volátil, os hidrocarbonetos terpênicos apresentam a fórmula molecular $\left(\mathrm{C}_{5} \mathrm{H}_{8}\right)_{\mathrm{n}}$, sendo classificados em monoterpenos os que apresentarem $\mathrm{n}$ igual 2 , sesquiterpenos para $\mathrm{n}$ 


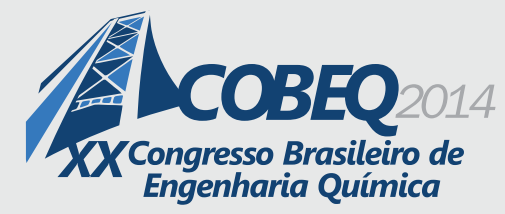

igual a 3 e diterpenos para $n$ igual a 4. Os compostos oxigenados, ou terpenóides, são derivados de terpenos, os quais podem conter o grupo hidroxila ou carbonila (Colecio-Juárez et al., 2012) e englobam várias classes de espécies químicas tais como aldeídos, cetonas, ésteres, álcoois e os ácidos (Gironi e Maschietti, 2012).

O óleo essencial de cravo (Eugenia caryophillata) possui diversas atividades biológicas tais como antibacteriana, antifúngica, antioxidante e, também, propriedades inseticidas. Em alimentos este óleo é utilizado como agente aromatizante e antimicrobiano (Wenqiang et al., 2007). Segundo Chaieb et al. (2006), os componentes majoritários do óleo de cravo são o eugenol, um álcool terpênico e o sesquiterpeno $\beta$-cariofileno.

Os compostos oxigenados assim como os hidrocarbonetos terpênicos apresentam aplicações industriais, no entanto, os terpenóides são os preferidos pelas indústrias devido suas propriedades aromáticas mais pronunciadas e pela elevada solubilidade em soluções aquosas (Cháfer et al., 2004). Os hidrocarbonetos terpênicos tendem a se decompor na presença de calor e oxigênio, gerando odores desagradáveis os quais podem contribuir para a perda de qualidade do óleo (Gironi e Maschietti, 2012).

Desta maneira, a redução do teor de hidrocarbonetos terpênicos e, consequentemente, a concentração do óleo em compostos oxigenados, prática industrial conhecida como desterpenação, pode ser realizada a fim de se melhorar a qualidade do óleo essencial bem como seu valor agregado (Sevgili et al., 2008).

Diversas metodologias têm sido propostas na literatura para o fracionamento/desterpenação de óleos essenciais. Entretanto, este processo apresenta algumas dificuldades devido às características delicadas dos referidos óleos (Arce et al., 2007). Neste sentido, a extração líquido-líquido tem sido citada como uma técnica promissora, principalmente devido à possibilidade de condução do processo a temperatura ambiente, o que seria interessante para a manutenção das propriedades sensoriais dos óleos (Haypek et al., 2000).

A utilização do etanol hidratado como solvente para a extração líquida além de visar uma maior segurança para a posterior utilização do extrato, seja para a aplicação deste em fármacos, cosméticos ou alimentos, também pode, dependendo da utilização, dispensar a etapa posterior de dessolventização uma vez que extratos alcoólicos de óleos essenciais apresentam elevada solubilidade em soluções aquosas, podendo, assim, ser facilmente adicionados a bebidas e perfumes. Ademais, estes extratos apresentam grande poder aromático e maior estabilidade devido ao fato das reações de oxidação serem reduzidas na presença de etanol (Li e Tamura, 2008).

O estudo do equilíbrio apresenta-se como a base para a consolidação da extração líquido-líquido aplicada ao fracionamento de óleos essenciais. Sendo assim, o objetivo deste trabalho foi o estudo sistematizado do equilíbrio de fases referente aos compostos comumente encontrados nos processos de fracionamento de óleo essencial de cravo por extração líquido-líquido, utilizando como solvente etanol hidratado. Dados experimentais de equilíbrio de fases, a $25,0 \pm 0,1{ }^{\circ} \mathrm{C}$, referentes a sistemas contendo componentes do óleo essencial de cravo ( $\beta$-cariofileno e eugenol) e solvente (etanol e água) 


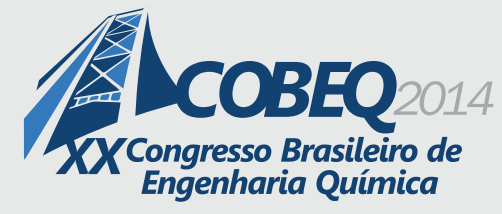

foram obtidos e correlacionados utilizando os modelos NRTL e UNIQUAC.

\section{MÉTODOS}

\subsection{Determinação experimental de dados de equilíbrio líquido-líquido}

Uma massa conhecida de eugenol (Sigma-Aldrich, $\geq 98 \%$, CAS 97-53-0) foi adicionada a uma massa conhecida de $\beta$-cariofileno (Sigma-Aldrich, $\geq 80 \%$, CAS 87-44-5). A esse óleo modelo, mistura do composto oxigenado e hidrocarboneto sesquiterpênico, foi adicionado o solvente etanol hidratado, na proporção 1:1 (óleo: solvente). As soluções etanólicas contendo 6, 12, 17, 23, 27, 32 e $40 \%$ de água foram preparadas pela adição de água deionizada (Millipore, modelo Direct-Q3, França) ao etanol absoluto (Merck, $\geq 99,8 \%$ ).

A concentração global da mistura foi previamente determinada e cada componente do sistema ( $\beta$-cariofileno + eugenol + etanol hidratado) foi pesado em balança analítica (precisão $0,0001 \mathrm{~g}$ ) (Adam, modelo PW 254, EUA) diretamente em tubo de polipropileno (capacidade 15 e $50 \mathrm{~mL}$ ).

Os tubos foram vedados e agitados vigorosamente por 10 minutos com auxílio de agitador (IKA, modelo Lab dancer, Alemanha), centrifugados por 30 minutos a 5000 g, na temperatura de 25 ${ }^{\circ} \mathrm{C}$ em centrífuga com controle de temperatura (Thermo Electron Corporation, modelo CR3i, França) e deixados em repouso durante 24 horas em banho termostatizado (Marconi, modelo MA-184, Brasil), a $25,0 \pm 0,1{ }^{\circ} \mathrm{C}$. Após o tratamento, amostras das fases foram retiradas e imediatamente submetidas às análises de cromatografia gasosa com detector de ionização de chama (CG-DIC, Shimadzu, modelo 2010AF, Japão) e titulação Karl Fischer (Metrohm, modelo 787 KF Titrino, Suíça) para determinação do teor de água.

As condições experimentais utilizadas no CG-DIC foram: coluna DBFFAP $0,25 \mu \mathrm{m}, 30 \mathrm{~m} \mathrm{x}$ $0,25 \mathrm{~mm}$ d.i. (Agilent, EUA); hélio como gás de arraste na taxa de $1,13 \mathrm{~mL} / \mathrm{min}$; temperatura do injetor de $250{ }^{\circ} \mathrm{C}$; temperatura da coluna de $(100$ a 240$){ }^{\circ} \mathrm{C}$ (taxa de $\left.8{ }^{\circ} \mathrm{C} / \mathrm{min}\right)$; temperatura do detector de $280^{\circ} \mathrm{C}$. Neste trabalho foi utilizada a metodologia de padronização externa.

\subsection{Modelagem dos dados experimentais de equilíbrio}

Dados experimentais de equilíbrio do sistema modelo de óleo de cravo composto por $\beta$ cariofileno (1) + eugenol (2) + etanol (3) + água (4) foram usados para ajustar parâmetros de interação dos modelos NRTL e UNIQUAC. As equações para os coeficientes de atividade de ambos os modelos, expressos em frações mássicas, podem ser visualizados em Rodrigues et al. (2004).

A estimativa dos valores dos parâmetros de interação binários foi baseada na minimização da função objetivo de composições (Equação 1), de acordo com Stragevitch e d'Ávila (1997).

$$
S=\sum_{m}^{D} \sum_{n}^{N} \sum_{i}^{C-1}\left[\left(\frac{w_{\text {inm }}^{F T, e x}-w_{\text {inm }}^{F T \text {,calc }}}{\sigma_{w_{\text {inm }}^{F T}}}\right)^{2}+\left(\frac{w_{\text {inm }}^{F S, e x}-w_{\text {inm }}^{F S, \text { calc }}}{\sigma_{w_{\text {inm }}^{F S}}}\right)^{2}\right]
$$




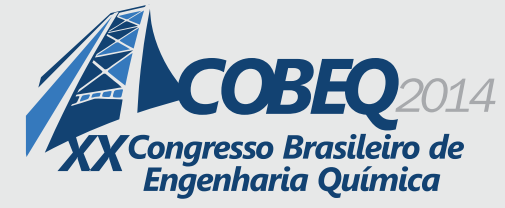

onde $D$ é o número total de grupos de dados, $N$ é o número total de linhas de amarração e $C$ é o número total de componentes no grupo de dados $m$. $w$ é fração mássica, os subescritos $i, n$ e $m$ são componente, tie line e número de grupos, respectivamente, e os superescritos $F T$ e $F S$ referem-se às fases terpênica e solvente, respectivamente; ex e calc referem-se aos valores experimentais e calculados das concentrações dos componentes nas fases. ${ }^{\sigma_{w_{i m m}^{F T}}} \mathrm{e}^{\sigma_{w_{i m m}^{F S}}}$ são os valores dos desvios padrão observados nas composições das duas fases líquidas.

Os desvios entre os valores das composições experimentais e calculadas foram estimados de acordo com a Equação 2.

$$
\Delta w=100 \sqrt{\frac{\sum_{n}^{N} \sum_{i}^{C}\left[\left(w_{i, n}^{F T, e x}-w_{i, n}^{F T, \text { calc }}\right)^{2}+\left(w_{i, n}^{F S, e x}-w_{i, n}^{F S, \text { calc }}\right)^{2}\right]}{2 N C}}
$$

\section{RESULTADOS E DISCUSSÃO}

A mistura modelo de óleo essencial de cravo foi composta por $\beta$-cariofileno e eugenol, os componentes majoritários do óleo segundo Chaieb et al. (2006). Os dados experimentais de equilíbrio obtidos, na temperatura de $25,0 \pm 0,1^{\circ} \mathrm{C}$, para o sistema modelo de óleo de cravo, composto por $\beta$ cariofileno (1) + eugenol (2) + etanol (3) + água (4), foram utilizados para ajustar os parâmetros de interação binários dos modelos termodinâmicos NRTL e UNIQUAC. Os parâmetros de interação binários obtidos para ambos os modelos são apresentados na Tabela 1, sendo os desvios entre as composições experimentais e calculadas, obtidos via Equação 2, mostrados na Tabela 2.

Tabela 1 - Parâmetros binários ajustados para o sistema composto por $\beta$-cariofileno (1), eugenol (2), etanol (3) e água (4), a $25,0 \pm 0,1^{\circ} \mathrm{C}$

\begin{tabular}{lllllll}
\hline \multirow{2}{*}{ Pares ij } & \multicolumn{3}{c}{ Modelo NRTL } & & \multicolumn{2}{c}{ Modelo UNIQUAC } \\
\cline { 2 - 4 } \cline { 6 - 7 } & $\mathrm{A}_{\mathrm{ij}}$ & $\mathrm{A}_{\mathrm{ji}}$ & $\mathrm{\alpha}_{\mathrm{ij}}$ & & $\mathrm{A}_{\mathrm{ij}}$ & $\mathrm{A}_{\mathrm{ji}}$ \\
\hline 12 & $-290,53$ & $-2,1529$ & 0,10001 & & $-78,717$ & 4,1555 \\
13 & 277,75 & 525,91 & 0,31879 & & 349,97 & $-95,189$ \\
14 & 229,38 & 5573,3 & 0,10542 & & 773,69 & 285,93 \\
23 & $-927,59$ & 552,42 & 0,10012 & & 100,33 & $-170,21$ \\
24 & $-779,61$ & 4771,1 & 0,15961 & & $-101,83$ & 275,00 \\
34 & 462,41 & $-704,95$ & 0,10001 & & 283,57 & $-320,04$ \\
\hline
\end{tabular}

O coeficiente de distribuição $\left(k_{i}\right)$ (Equação 3) é a razão entre as frações mássicas de cada um dos componentes presentes na fases solvente e terpênica.

$$
k_{i}=\frac{w_{i}^{F S}}{w_{i}^{F T}}
$$




\section{Acoser XCongresso Brasileiro de Engenharia Química}

19 a 22 de outubro de 2014

Florianópolis/SC

Tabela 2 - Desvios médios entre as composições experimentais e calculadas para o sistema modelo de óleo de cravo

\begin{tabular}{ccc}
\hline $\begin{array}{l}\text { Teor nominal de água no solvente }(\% \\
\text { mássica) }\end{array}$ & $\Delta \mathrm{w}(\%)$ NRTL & $\Delta \mathrm{w}(\%)$ UNIQUAC \\
\hline 6 & 0,80 & 0,47 \\
12 & 0,92 & 0,84 \\
17 & 0,31 & 0,51 \\
23 & 0,44 & 0,44 \\
27 & 0,37 & 0,34 \\
32 & 0,22 & 0,33 \\
40 & 0,28 & 0,29 \\
Desvio global & 0,51 & 0,50 \\
\hline
\end{tabular}

Os valores de coeficiente de distribuição dos compostos $\beta$-cariofileno $\left(k_{1}\right)$ e eugenol $\left(k_{2}\right)$ em função do teor de compostos oxigenados na fase terpênica para os sistemas modelo de óleo essencial de cravo são apresentados na Figura 1. Nesta figura é possível observar que, para todos os níveis de água no solvente, o aumento do teor de compostos oxigenados na fase terpênica diminui a extração do eugenol. No que se refere ao coeficiente de distribuição do $\beta$-cariofileno, este parece não sofrer mudanças significativas com relação a modificações na composição de eugenol na fase terpênica.
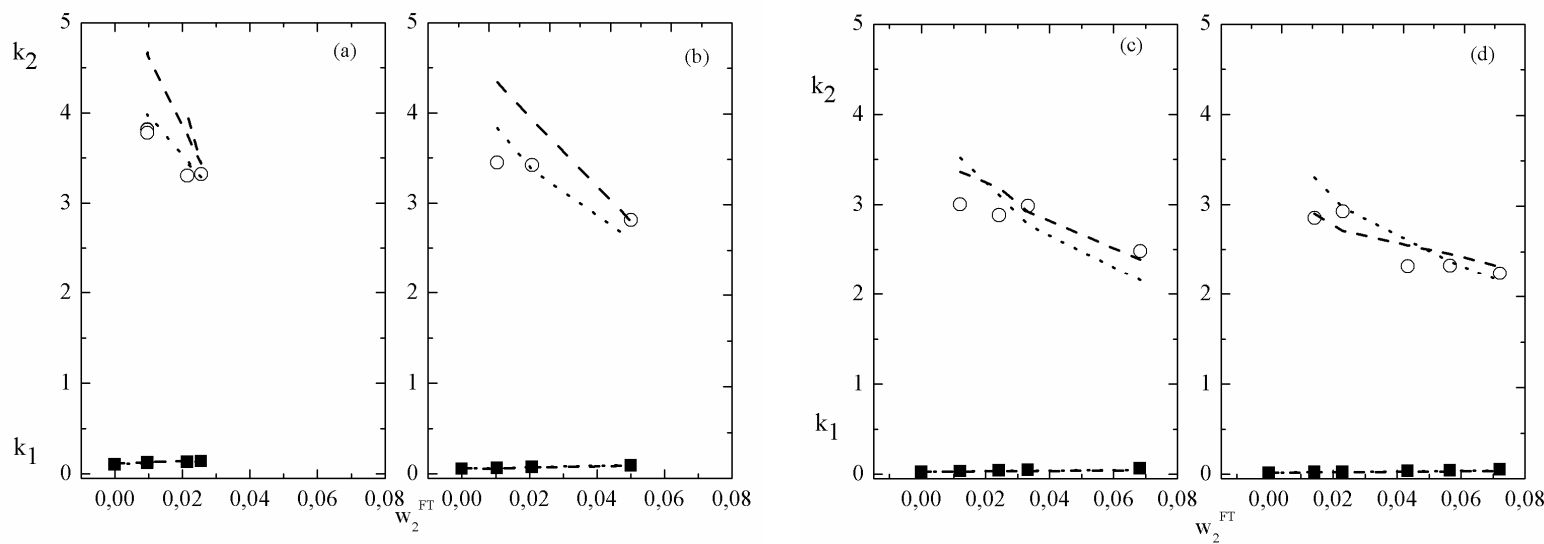

Figura 1 - Coeficientes de distribuição, a $25,0 \pm 0,1^{\circ} \mathrm{C}$, do $\beta$-cariofileno $\left(\mathrm{k}_{1}\right)$ e eugenol $\left(\mathrm{k}_{2}\right)$ em função da fração mássica de eugenol na fase terpênica $\left(\mathrm{w}_{2}{ }^{\mathrm{FT}}\right)$. (a) etanol com 12,46 \% de água, em massa; (b) etanol com 16,64 \% de água, em massa; (c) etanol com 23,60\% de água, em massa; (d) etanol com 27,99 \% de água, em massa. Experimental: (ロ) $\mathrm{k}_{1}$ e (o) $\mathrm{k}_{2}$. Calculado: (---) NRTL e (.....) UNIQUAC.

O aumento da hidratação do solvente está associado à diminuição da solubilidade mútua levando à diminuição dos coeficientes de partição dos componentes do óleo essencial, em maior extensão para o eugenol do que para o $\beta$-cariofileno (vide Figura 1). Adicionalmente, verifica-se que, entre os componentes do óleo essencial de cravo modelo, o composto oxigenado é o que apresenta os maiores valores de coeficiente de partição, possibilitando desta maneira, o fracionamento da mistura.

A seletividade do solvente (Equação 4), infere sobre a capacidade do solvente em extrair 


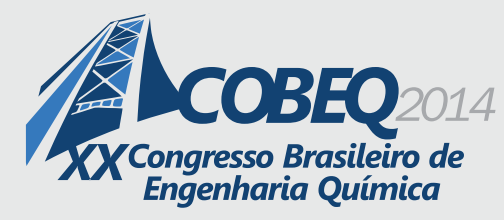

19 a 22 de outubro de 2014

Florianópolis/SC

compostos oxigenados sem extrair teores apreciáveis de hidrocarboneto sesquiterpênico.

$$
S_{i / j}=\frac{k_{i}}{k_{j}}
$$

Os valores de seletividade, a $25,0 \pm 0,1{ }^{\circ} \mathrm{C}$, do eugenol em relação ao $\beta$-cariofileno $\left(\mathrm{S}_{2 / 1}\right)$ em função do teor de eugenol na fase terpênica (FT) são mostrados na Figura 2, na qual observa-se valores de seletividade mais elevados associados a maior hidratação do solvente. De fato, a inclusão do componente água faz-se imprescindível para a formação de duas fases líquidas, uma vez que o etanol é completamente miscível nos componentes do óleo essencial. A água modifica a polaridade do solvente tornando o processo factível.

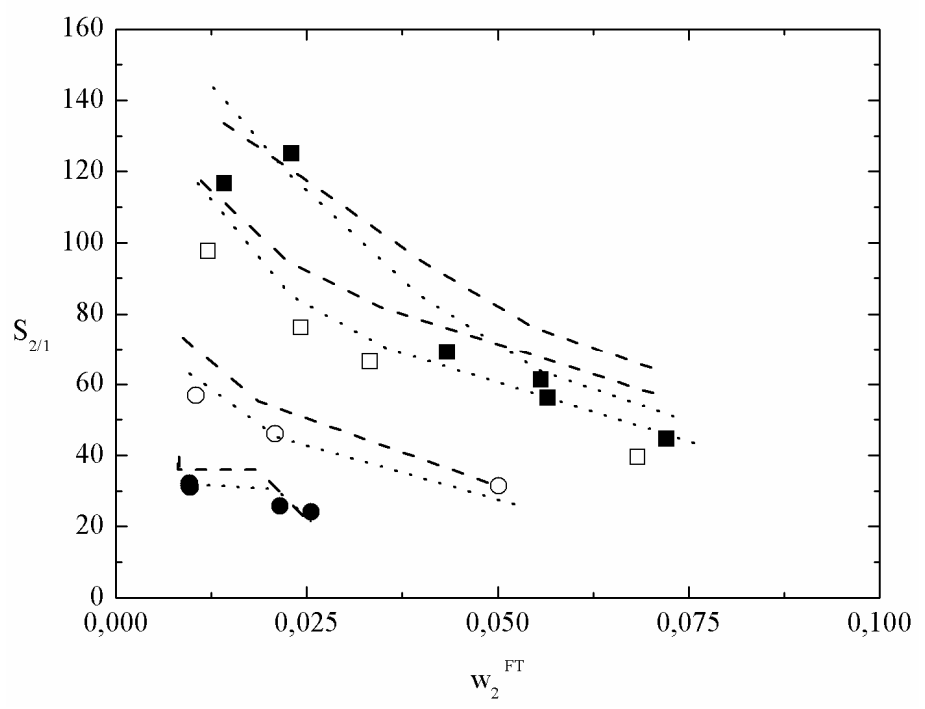

Figura 2 - Seletividade do eugenol em relação ao $\beta$-cariofileno $\left(\mathrm{S}_{2 / 1}\right)$ em função da fração mássica de eugenol na fase terpênica $\left(\mathrm{w}_{2}{ }_{\mathrm{FT}}\right)$. Experimental: $(\bullet)$ etanol com 12,46\% de água; ( $\circ$ ) solvente etanol com 16,64 \% de água; ( $\square$ ) etanol com $23,60 \%$ de água e (ロ) etanol com 27,99 \% de água. Calculado: (---) NRTL e (...) UNIQUAC.

Na Figura 2 verifica-se, ainda, que os valores de seletividade são muito superiores a unidade, ou seja, o solvente apresenta maior afinidade pelo eugenol do que pelo $\beta$-cariofileno sugerindo, assim, a possibilidade do fracionamento do óleo essencial de cravo.

Alguns trabalhos na literatura reportam o equilíbrio líquido-líquido de compostos comumente encontrados em óleos essenciais e solvente etanol hidratado (Koshima et al., 2012; Capellini et al., 2012; Chiyoda et. al., 2011; Arce et al., 2004, 2005). Os dados experimentais apresentados para o sistema modelo de óleo de cravo estão qualitativamente de acordo com estes estudos, entretanto, quantitativamente verifica-se que os valores de coeficiente de distribuição do composto oxigenado bem como de seletividade apresentam-se superiores. Por outro lado, observa-se que o hidrocarboneto sesquiterpênico $\beta$-cariofileno apresenta uma menor partição em relação ao hidrocarboneto 


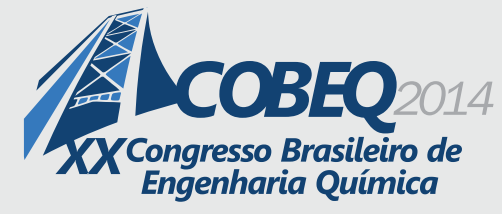

monoterpênico limoneno.

Com base nas Figuras 1 e 2 apresentadas, as quais possibilitam confrontar os valores experimentais determinados com os calculados pelos modelos NRTL e UNIQUAC, é possível inferir que ambos os modelos proporcionam uma descrição satisfatória e semelhante dos dados experimentais de coeficiente de partição e seletividade. Estes comentários estão de acordo com os baixos valores de desvio global obtidos para os modelos termodinâmicos (vide Tabela 2).

\section{CONCLUSÕES}

Pode-se inferir que o processo de desterpenação do óleo essencial de cravo, utilizando como solvente o etanol hidratado é viável, sendo possível a obtenção de frações enriquecidas em eugenol. Em comparação com os dados encontrados na literatura, estes sistemas apresentaram valores superiores de seletividade e coeficientes de partição dos terpenóides, fatores que viabilizam a eficiência do processo. Com relação à descrição dos dados experimentais, os modelos estudados apresentaram desempenho similar e satisfatório com valores de desvio global próximos a $0,5 \%$. A estimativa dos parâmetros de interação binários é importante para predizer o equilíbrio de fases envolvendo sistemas reais e simular o processo de fracionamento.

\section{AGRADECIMENTOS}

Os autores agradecem à FAPESP (Fundação de Amparo à Pesquisa do Estado de São Paulo, processos n ${ }^{\circ}$ 2010/20789-0; 2011/02476-7; 2012/15323-7; 2012/15317-7) e CNPq (bolsa PIBIC) pelo apoio financeiro.

\section{REFERÊNCIAS}

ARCE A., POBUDKOWSKA A., RODRÍGUEZ O., SOTO A. Citrus essential oil terpenless by extraction using 1-ethyl-3-methylimidazolium ethylsulfate ionic liquid: effect of the temperature. Chem. Eng. J., v.133, p. 213-218, 2007.

ARCE, A., MARChIARO, A., MARTÍNEZ-AGEITOS, J. M., SOTO, A. Citrus essential oil deterpenation by liquid-liquid extraction. Can. J. Chem. Eng., v. 83, p.366-370, 2005.

ARCE, A., MARCHIARO, A., SOTO, A. Liquid-liquid equilibria of linalool + ethanol + water, water + ethanol + limonene, and limonene + linalool + water systems. J. Solution Chem., v. 33, p. 561-569, 2004.

BIZZO, H. R.; HOVELL, A. M. C.; REZENDE, C. M. Óleos essenciais no Brasil: aspectos gerais, desenvolvimento e perspectivas. Química Nova, v.32, p.588-594, 2009.

CAPELlini, M. C., CARVAlHO, F. H., CHIYODA, C., ARACAVA, K. K., RODRIGUES, C. E. C. Estudo do equilíbrio líquido-líquido e modelagem termodinâmica de sistemas modelo compostos por componentes do óleo essencial de orégano e solvente alcoólico, a $25^{\circ} \mathrm{C}$. XIX COBEQ, 2012.

CHÁFER, A.; MUÑOZ, R.; BURGUET, M. C.; BERNA, A. The influence of the temperature on the Liquid-liquid equilibria of the mixture linalool + ethanol $+\mathrm{H}_{2} \mathrm{O}$. Fluid Phase Equilib, v.224, p.251- 


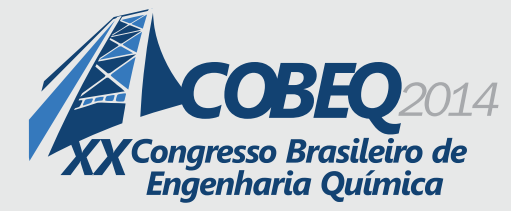

256, 2004.

CHAIEB, K., HAJLAOUI, H., ZMANTAR, T., KAHLA-NAKBI, A. B., ROUABHIA, M., MAHDOUANI, K., BAKHROUF, A. The chemical composition and biological activity of clove essencial oil, Eugenia caryophyllata (Syzigium aromaticum L. Myrtaceae): a short review. Phytother. Res., v.21, p. 501-506, 2006.

CHIYODA, C., CAPELliNI, M. C., GEREMIAS, I. M., CARVALHO, F. H., ARACAVA, K. K., BUENO, R. S., GONÇALVES, C. B., RODRIGUES. C. E. C. Deterpenation of bergamot essential oil using liquid-liquid. J. Chem. Eng. Data, v.56, p. 2362-2370, 2011.

COLECIO-JUÁREZ, M. C., RUBIO-NÚÑEZ, R. E., BOTELLO-ÁLVAREZ, J. E., MARTÍNEZGONZÁLEZ, G. M., NAVARRETE-BOLAÑOS, J. L., JIMÉNEZ-ISLAS, H. Characterization of volatile compounds in the essential oil of sweet lime (Citrus limetta Risso). Chil. J. Agr. Res., v.72, p. 275-280, 2012.

DIAZ, S.; ESPINOSA, S.; BRIGNOLE, E.A. Citrus peel oil deterpenation with supercritical fluids. Optimal process and solvent cycle design. Journal of Supercritical Fluids, v.35, p.49-61, 2005.

GIRONI, F., MASCHIETTI, M. Phase equilibrium of the system supercritical carbon dioxide-lemon essential oil: New experimental data and thermodynamic modeling. J. Supercrit. Fluids, v.70, p.8-16, 2012.

HAYPEK， E.; SILVA， L.H.M.; BATISTA， E.; MARQUES， D. S.; MEIRELES， M.A.A.; MEIRELLES, A.J.A. Recovery of aroma compounds from orange essential oil, Braz. J. Chem.l Eng., v.17, p.705-712, 2000.

KOSHIMA, C. C., CAPELlini, M. C., GEREMiAS, I. M., ARACAVA, K. K., GONÇALVES, C. B., RODRIGUES. C. E. C. Fractionation of lemon essential oil by solvente extration: phase equilibrium for model systems at T=298.2 K. J. Chem. Thermodyn, v.54, p. $316-321,2012$.

LI, H.; TAMURA, K. Ternary liquid-liquid equilibria for (water + terpene + 1-propanol or 1-butanol) systems at the temperature 298.15K. Fluid Phase Equilib., v.263, p.223-230, 2008.

RODRIGUES, C. E. C.; PESSÔA FILHO, P. A.; MEIRELLES, A. J. A. Phase Equilibrium for the System Rice Bran Oil + Fatty Acids + Ethanol + Water + g-Oryzanol + Tocols. Fluid Phase Equilib., v. 216, p. 271-283, 2004.

SEVGILI, L. M.; SAHIN, S.; KIRBASLAR, S. I. Liquid-liquid equilibria of (limonene + linalool + ethylene glycol or diethylene glycol or triethylene glycol or 1,2-propylene glycol) ternary systems, $J$. Chem. Eng. Data, v.53, p.737-741, 2008.

STRAGEVITCH, L., D'AVILA, S. G. Application of a generalized maximum likelihood method in the reduction of multicomponent liquid-liquid equilibrium data. Braz. J. Chem.l Eng., v.14, p.41-52, 1997.

WENQIANG, G., SHUFEN, L., RUIXIANG, Y., SHAOKUN, T., CAN, Q. Comparison of essential oils of clove buds extracted with supercritical carbon dioxide and other three traditional extraction methods. Food Chem., v.101, p.1558-1564, 2007. 